\title{
Errata
}

M.E. Newton, D.I. Southern and R.J. Wood: Relative DNA Content of Normal and Sex-Ratio Distorting Spermatozoa of the Mosquito, Aedes aegypti (L.). Chromosoma (Berl.) 67, 253-261 (1978)

\section{Page 257}

Legend, line 1 read $M+/ m+$ Text line $15 \mathrm{read} M+/ m+$

Page 260

References, line 3 read 1965 instead of $m+/ m+$ instead of $m+/ m+$

instead of 1905 